\title{
Sara Bischetti
}

\section{Il punto sui manoscritti dei Documenti d'Amore}

\begin{abstract}
Il contributo si pone l'obiettivo di analizzare i due testimoni principali dei Documenti d'Amore di Francesco da Barberino, ovvero il Barb. lat. 4076 e il Barb. lat. 4077 conservati presso la Biblioteca Apostolica Vaticana, mediante una nuova e approfondita indagine codicologica e paleografica. L'esame comparativo tra i due esemplari ha permesso non solo di avvalorare l'ipotesi di autografia totale per il Barb. lat. 4076, e di autografia parziale per il Barb. lat. 4077, ma anche di riscostruirne le modalità di allestimento, nonché i rapporti temporali intercorrenti tra i due. Questi elementi hanno inoltre consentito di approfondire la questione dell'autografia autoriale, legata alla più ampia tematica del libro d'autore trecentesco.
\end{abstract}

This contribution presents a detailed analysis of the two main witnesses of Francesco da Barberino's Documenta Amoris (Barb. lat. 4076 and Barb. lat. 4077, Biblioteca Apostolica Vaticana), based on an in-depth codicological and palaeographical study. It not only confirms the hypothesis that Francesco himself wrote all of Barb. lat. 4076 and parts of Barb. lat. 4077, but also helps to reconstruct details of the writing process and the chronological relationship between the two witnesses. The findings shed new light on the question of authorial authorship, which is linked to the broader topic of the fourteenth-century libro d'autore.

Parole chiave: Francesco da Barberino, manoscritti, autografia, libro d'autore

\section{Premessa}

Il progetto di una edizione critica digitale dei Documenti d'Amore di Francesco da Barberino, basata sul testo trasmesso nella sua interezza dal noto manoscritto della Biblioteca Vaticana, Barb. lat. 4076, ha presupposto come lavoro preliminare e imprescindibile una diretta e rinnovata indagine codicologica e paleografica dell'esemplare. ${ }^{1}$ A questa prima ricerca ha fatto seguito quella re-

1 L'edizione digitale del testo, coordinata da Antonio Montefusco e da Tiziana Mancinelli, è tuttora in corso di svolgimento e si inserisce all'interno del progetto Biflow - Bilingualism in Florentine and Tuscan works, ca. 1260-ca. 1430, (ERC Starting Grant 2014- 637533); vedi, in questo stesso volume, il contributo di Tiziana Mancinelli alle pp. 65-89. 
lativa al codice "gemello", il Barb. lat. 4077, entrambe presunte "copie d'autore”, sulla cui parziale o totale autografia (sia grafica che iconica), e sullo stretto rapporto che li lega, si discuterà approfonditamente nel corso del presente contributo, cercando anche di avanzare ipotesi che provino a far luce su alcune questioni, che allo stato attuale delle ricerche rimangono ancora aperte e discordanti. ${ }^{2}$ Lo scopo precipuo è stato dunque quello di approfondire innanzitutto gli aspetti codicologici e grafici dei due testimoni, mediante un approccio che ha previsto più livelli di analisi, e che ha consentito una indagine delle relazioni intercorrenti tra i differenti blocchi testuali (versi poetici in volgare, translatio latina e glosse in latino), e tra questi e il ricco apparato decorativo che, come si vedrà, assume un ruolo significativo, perché anch'esso finalizzato - analogamente agli altri elementi grafico-visivi a rendere esplicite le connessioni semantiche che permeano la complessa compagine strutturale dell'insieme. Tutto ciò in previsione di quello che si auspica possa essere, in termini di "intertestualità" e di configurazione dinamica dei contenuti, il progetto di edizione digitale dell'opera, il solo che possa tentare di ricreare, almeno visivamente, e attraverso la realizzazione di un modello organizzativo costruito ad hoc, il disegno programmatico dell'autore.

2 Per una panoramica sulle ipotesi finora esposte a tal riguardo cfr. almeno Egidi, Le miniature dei codici Barberiniani, pp. 1-20, 78-95; Francesco da Barberino, I Documenti d'Amore (ed. Egidi); Goldin, Testo e immagine, pp. 125-138; Petrucci, Minuta, autografo, libro d'autore, pp. 398-414, in partic. pp. 409-410; Id., Scrivere il testo, pp. 209-227, in partic. pp. 224-225; Id., Modello notarile e testualità, pp. 138-139; Id., La scrittura del testo, pp. 289-290; Id., Minima barberina, pp. 1005-1009; Id., Storia e geografia delle culture scritte, pp. 1226-1227; Id., Dalla minuta al manoscritto d'autore, pp. 353-372, in partic. pp. 364-365; Panzera, Per l'edizione critica, pp. 91-118; Supino Martini, Per la tradizione manoscritta, pp. 945-954. Per le ipotesi relative all'autografia barberiniana nei disegni presenti nel Barb. lat. $4077 \mathrm{cfr}$. per primo Egidi, Le miniature dei codici Barberiniani e Francesco da Barberino, I «Documenti d'Amore» (ed. Egidi), IV, pp. XXIII-XXVIII, poi Panzera, Per l'edizione critica, p. 97, n. 16. Di parere differente Pächt, Der Weg von der zeichnerischen, pp. 178-184, in partic. pp. 183-184, e la stessa Supino Martini, Per la tradizione manoscritta, pp. 947-950, che limita la responsabilità del Barberino ai soli disegni preparatori per gli esemplari destinati alla pubblicazione; vedi, a tal proposito, anche Ciccuto, Francesco da Barberino e il suo Bildercodex, pp. 83-95. 


\section{L’analisi codicologica e paleografica del Barb. lat. 4076}

Una dettagliata descrizione dei due manoscritti barberiniani è già stata fornita da Armando Petrucci, ${ }^{3}$ che ha ampliato quella contenuta nell'ultimo dei quattro volumi dell'edizione diplomatica condotta nell'arco di un ventennio da Francesco Egidi, ancora oggi punto di riferimento imprescindibile per lo studio del testo. ${ }^{4}$ Occorre, tuttavia, segnalare alcune discrepanze a livello codicologico che il nuovo esame de visu ha permesso di portare alla luce, in primo luogo per quel che concerne la struttura fascicolare del Barb. lat. 4076 che, come si diceva, rappresenta il testimone completo dei Documenti d'Amore. L'irregolarità della fascicolazione fu sostenuta per primo da Petrucci che ne suddivideva così l'assetto: 1 carta +3 fascicoli di nove carte +5 ternioni +1 binione +2 quaternioni, preceduti da otto carte iniziali aggiunte in un momento coevo, ma non contestuale alla copia, e contenenti l'Index rerum vergato su tre colonne di scrittura da una calligrafica mano italiana della metà del secolo XIV. Di contro, Maria Cristina Panzera, tornata sull'argomento qualche anno più tardi, rilevava una struttura fascicolare suddivisa in otto ternioni, oltre al quaternione iniziale. ${ }^{5}$ Solo di recente, in occasione della nuova edizione critica del testo, Marco Albertazzi ha proposto una distribuzione fascicolare comprensiva di entrambe le suddivisioni esposte in precedenza. ${ }^{6} \mathrm{Ci}$ troviamo dunque davanti non solo a pareri discordanti, ma anche ad analisi fascicolari che in tutti i casi non risultano essere compatibili con il numero delle carte di cui si compone il manoscritto, essendo l'esemplare Barberiniano formato da 102 carte, consistenza, tra l'altro, dichiarata dagli studiosi stessi (cfr. Tab. 1).

Tabella 1: Struttura fascicolare del Barb. lat. 4076.

\begin{tabular}{lll}
\hline Petrucci & Panzera & Albertazzi \\
\hline 1 carta +3 fascicoli di nove carte +5 & 8 ternioni $=$ & 1 carta +3 fascicoli di nove \\
ternioni +1 binione +2 quaternioni $=$ & consistenza & $\begin{array}{l}\text { carte }+5 \text { ternioni }+1 \text { binione }+ \\
\text { consistenza cc. } 76\end{array}$ \\
& cc. 48 & $\begin{array}{l}2 \text { quaternioni }+8 \text { ternioni }= \\
\text { consistenza totale cc. } 94\end{array}$ \\
\hline
\end{tabular}

3 Cfr. Petrucci, Minima barberina, p. 1006.

4 Francesco da Barberino, I Documenti d'Amore (ed. Egidi), pp. XVI-XXIII.

5 Panzera, Per l'edizione critica, p. 93.

6 Francesco da Barberino, I Documenti d'Amore, I (ed. Albertazzi), p. IX. Lo studioso parla di: 1 foglio e 3 fascicoli di 9 fogli +1 duerno +2 quaderni + otto fascicoli ternioni. 
L'esame autoptico del codice ha consentito invece di rilevare una struttura fascicolare del tutto regolare (del resto già sostenuta dall'Egidi), ${ }^{7}$ composta da 17 fascicoli ternioni, ai quali sono premesse - e in questo mi trovo d'accordo con quanto sostenuto finora - le prime otto carte non facenti parte dell'assetto originario del manufatto, cartulate da mano moderna in numeri romani, che mostrano una pergamena di colore e spessore diverso da quella utilizzata per i restanti fascicoli. Tale suddivisione viene oltretutto confermata dalla presenza costante dei richiami posizionati al centro del margine inferiore dell'ultima carta verso dei fascicoli. La regolarità dell'impianto fascicolare d'altronde non stupisce, ed è in perfetta sintonia con la progettualità organizzativa dell'insieme, dove nulla viene lasciato al caso, e dove la complessa pianificazione formale dell'allestimento pare essere preventivamente pensata in ogni suo aspetto. Basti osservare, in primo luogo, la particolare e articolata mise en page che inquadra il testo su tutti e quattro i lati, secondo una prassi in uso nel manoscritto giuridico (bolognese in particolare), e in quello universitario a doppio apparato esegetico, con il testo poetico in volgare al centro della pagina (disposto su una o su due colonne di scrittura), la traduzione latina ai lati (in prosa), e la glossa, anch'essa in latino, che incornicia tutt'intorno i primi due "strati” testuali, in una calibrata impaginazione che potremmo definire a "corone concentriche”, ove il contenuto in volgare viene messo in risalto dagli altri due blocchi testuali (es. c. 3r; Fig. 2). Il riempimento dello spazio a disposizione è gestito in modo uniforme per tutto il codice, e l'effetto estetico finale risulta perfettamente omogeneo nelle due facciate delle pagine affrontate (recto/verso); si nota, infatti, una specularità nell'impostazione del commento, esteso su uno spazio più piccolo nella parte adiacente ai margini interni (cc. 2v-3r; Fig. 2). Della complessa mise en page fa parte integrante anche il pregevole apparato decorativo che consta di ben 27 miniature di alto livello esecutivo, di argomento allegorico e tecnico-scientifico, strettamente collegate alla componente testuale: il testo sembra infatti fungere da didascalia all'immagine che, trovandosi all'inizio di ogni Documento, fa da introduzione a questo; ma a sua volta la componente decorativa diventa essa stessa didascalia in una «sorta di procedimento circolare che dal testo porta all'immagine e dall'immagine torna al testo», ${ }^{8}$ con le glosse che forniscono una chiave di lettura per interpretare le miniature, rendendo evidente l'imprescindibile complementarità dell'insieme (c. 64r; Fig. 9). Il linguaggio visivo, dotato già di per sé di significato (tanto da assurgere al valore di "linguaggio verbale"), contribuisce a comprendere meglio e sotto certi aspetti è il solo in grado di farlo - concetti astratti espressi dalla com-

7 Francesco da Barberino, I Documenti d'Amore (ed. Egidi), p. XVII.

8 Goldin, Testo e immagine, p. 127. 
ponente letteraria in una specie di reciproca cooperazione tra scriptura e pictura. ${ }^{9}$ Tale rapporto è stato ulteriormente messo in risalto dall'esame codicologico che ha altresì evidenziato il carattere semantico che pervade l'intera compagine strutturale, caratterizzata da legami intrinseci tra la componente verbale e i suoi diversi “corredi” (illustrativi, paragrafici e peri-grafici), non solo in termini di interazioni spaziali ma anche, e ad un livello più alto, di significato. Se ad esempio si pone l'attenzione sul sistema delle rubriche, oppure sull'utilizzo degli inchiostri, si può notare una volontà progettuale a priori volta a tradurre visivamente il messaggio verbale, in una sorta di completa, e complessa, combinazione tra ogni elemento del codice. Per quel che riguarda le rubriche, i versi del testo in volgare (ordinati a gruppi di due o di quattro a seconda della disposizione, rispettivamente, ad una o a due colonne) sono individuati dall'iniziale maiuscola al tratto, toccata di rosso, e talvolta da un piccolo segno di paragrafo, e segnalati in margine da "graffe" che li includono; inoltre, ogni incipit è a sua volta individuato da un'iniziale calligrafica semplice, tracciata nello stesso inchiostro del testo, ma a doppia filettatura ed entro riquadro, e dalla rubrica esplicativa. Analoghe iniziali si ritrovano in maniera speculare nell'auto-traduzione latina, in corrispondenza dei paragrafi relativi. Riguardo all'uso degli inchiostri, invece, ho notato una differenza di colore per i diversi blocchi testuali che varia all'interno del manoscritto dal marrone chiaro al bruno. Generalmente, il testo della glossa è in inchiostro più chiaro rispetto a quello adoperato per il testo volgare e per la traduzione latina; quello più scuro sembra invece caratterizzare in misura maggiore, ma non costantemente, la translatio in latino, anche se in alcune carte mi pare si possa notare l'uso di un inchiostro analogo per testo volgare e latino. Alle lievi differenze riscontrate nel colore degli inchiostri fa riscontro l'uso di un diverso strumento scrittorio: penna a punta mozza per i versi centrali e la traduzione, ma tagliata in modo differente, poiché nel primo caso il tracciato appare meno marcato del secondo; e penna a punta fina

9 Del rapporto intrinseco che lega le immagini al testo, e viceversa, parla approfonditamente già Egidi, Le miniature dei codici Barberiniani, pp. 5-6, sottolineando l'importanza da sempre conferita dal Barberino alla componente figurativa (soprattutto al disegno), e al suo ruolo semantico; vedi anche Goldin, Testo e immagine. In particolare, per l'influenza sull'autore dei Canzonieri provenzali iconizzati, diffusi nell'Italia del nord nel primo Trecento, cfr. Ciccuto, Icone della parola, pp. 13 sgg; e Id., Guinizzelli e Guittone, Barberino e Petrarca, pp. 77-87. Sul legame imprescindibile che lega il testo alle immagini anche in altre opere dell'autore, come il Reggimento e costumi di donna e l'Officiolo, vedi Giunti, L'interazione fra testo e immagini, pp. 121-144; Ead., «Né parlerai rimato», pp. 71-111; Sutton, The lost 'Officiolum', pp. 152-164; Bertolo, Apocalissi figurata, pp. 55-64; Bertolo, Nocita, L'Officiolum' ritrovato di $F$. da B., pp. 106-117; in questo studio gli studiosi mettono in luce il ruolo predominante della componente iconografica su quella testuale, alla quale è riservato uno spazio di gran lunga minore rispetto ai riquadri ospitanti le miniature. 
per la glossa, come dimostrerebbe il limitato contrasto della scrittura. L'adozione di inchiostri e di penne differenti, grazie alle quali mutano il modulo della scrittura, il tratteggio e il tracciato, sono chiaramente ulteriori espedienti per diversificare le partizioni testuali e per rendere immediatamente visibile la distribuzione a tre livelli del testo. Ogni elemento concorre a ciò: non solo la particolare mise en page, e dunque gli inchiostri, ma prima di tutto l'adozione di tre grafie apparentemente (perché volutamente) differenti nella facies complessiva (modulo, tracciato, morfologia). La scrittura del testo centrale in volgare si mostra infatti come una semigotica, ibridata di elementi cancellereschi, elegante e calligrafica, slanciata, ben spaziata, e sottile; quella della traduzione latina sembra invece accentuare $\mathrm{i}$ caratteri propri della textualis, presentandosi posata, diritta, serrata e compressa, con un tracciato leggermente contrastato e chiaroscurato; la scrittura della glossa è simile a quella del testo volgare, ma - analogamente ad altre «scripturae noturalis» ${ }^{10}$ - appare caratterizzata da un ductus più corsivo, da un modulo visibilmente più piccolo, e da un tracciato sottile.

Premesso ciò, e sulla base dei dati desunti dalla rinnovata indagine paleografica, vorrei tentare di formulare considerazioni più approfondite, e per certi versi distanti dalle ipotesi avanzate finora, sia relativamente all'identificazione delle mani che sono intervenute nel lavoro di trascrizione, sia riguardo al sistema grafico di appartenenza delle scritture adoperate, alle quali si lega - va da sé - la questione dell'autografia autoriale. Lasciando per il momento in sospeso tale problematica, vorrei innanzitutto accennare alle posizioni assunte sino ad oggi dalla critica per quel che concerne il responsabile o i responsabili della copia. Armando Petrucci, in opposizione alla tesi avanzata dall'Egidi sulla presenza di un'unica mano artefice dell'intero manoscritto, ${ }^{11}$ sosteneva l'analogia grafica tra la sola scrittura della traduzione latina e quella della glossa, adducendo quale prova identificante la caratteristica $s$ tonda finale con il tratto superiore orizzontale prolungato, visibile in entrambe le esecuzioni grafiche. ${ }^{12}$ Di contro, Paola Supino Martini proponeva un accostamento tra la scrittura del testo in volgare e quella del commento, riconducendo entrambe le realizzazioni

10 Riprendo in uso un termine adoperato da Armando Petrucci ormai in anni lontani, ma pur sempre valido, per indicare la scrittura tipica della glossa letteraria, vero e proprio genere letterario che si diffuse capillarmente nel corso del tardo-medioevo (ma che affondava nel sue radici nella tarda antichità) tra i letterati e i dotti dell'epoca, primo fra tutti Francesco Petrarca; a tal proposito cfr. Petrucci, La scrittura di Francesco Petrarca, in particolare le pp. 31-57; vedi anche Id., Libro e scrittura in Francesco Petrarca, pp. 5-20, in partic. p. 15.

11 Cfr. Francesco da Barberino, I Documenti d'Amore (ed. Egidi), IV, p. 20. Egidi riprende un'ipotesi già sostenuta nel Seicento da Ubaldini, Documenti d'Amore.

12 Petrucci, Minima barberina, p. 323. 
grafiche non all'area della gotica, come da sempre sostenuto, bensì a quella della corsiva cancelleresca ${ }^{13}$ (Tab. 2).

Tabella 2: Individuazioni delle mani intervenute nel Barb. lat. 4076.

\begin{tabular}{lll}
\hline Egidi & Petrucci & Supino Martini \\
\hline unica mano: & due mani: & due mani: \\
$\cdot$ mano A: testo italiano, & $\cdot$ mano A: testo italiano + & $\cdot$ mano A: testo italiano e \\
translatio latina e glossa & cc. $99 \mathrm{v}-101 \mathrm{v}$ & glossa \\
& $\cdot$ mano B: translatio latina & $\cdot$ mano B: translatio latina \\
& e glossa & \\
\hline
\end{tabular}

Sulla base delle ipotesi proposte finora sarei tentata di effettuare una operazione di raccordo tra la tesi di Petrucci e quella di Supino Martini, e di avvalorare così l'ipotesi iniziale suggerita da Egidi. Le analogie morfologiche riscontrabili, a mio parere, in tutti e tre i "livelli" grafici, permettono di supporre che il Barb. lat. 4076 sia stato in realtà vergato da un solo copista che adopera grafie differenti (almeno nell'aspetto) con la volontà consapevole di diversificare i tre blocchi testuali. ${ }^{14}$ Tra le lettere caratteristiche che permettono di accostare le tre esecuzioni si osservano: la $d$ con asta alta slanciata e uncinata, la $g$ a mo' di 9, con l'occhiello inferiore ovale e leggermente inclinato a sinistra, per lo più aperto, costante nel testo italiano e nelle glosse, ma che si ritrova, seppure in maniera più sporadica, anche nella parafrasi latina, dove è affiancata, in modo più assiduo, da una $g$ di tipo testuale, a forma di $s$ tagliata da un sottile tratto trasversale (es. cc. 42r, 69r; Figg. 8 e 11); nonostante si tratti di un elemento all'apparenza differenziante, in realtà, se si esaminano attentamente i versi poetici in volgare si può notare che essa appare identica in alcuni esempi interni al testo e nelle rubriche, dove la $g$ viene utilizzata in entrambe le forme (c. 42r; Fig. 8). E ancora: la $s$ tonda, finale di parola, a mo' di 6 o di 8, che Petrucci considera elemento caratterizzante per ipotizzare l'identità di mano tra parafrasi latina e glosse, si ritrova

13 Supino Martini, Per la storia della semigotica, pp. 249-264, in partic. pp. 253-254; la studiosa rivela la presenza, a partire dai primi anni del sec. XIV, di numerose scritture accumunate da elementi similari, e definibili con il termine di semigotica; esse sono, come afferma la studiosa, «di solito eseguite secondo un tracciato frazionato, ovvero, come le librarie, per successivi tocchi di penna, rivelano, tuttavia, una più o meno stretta connessione con la cancelleresca o comunque con un tracciato posato della corsiva» (cit. p. 251); cfr. anche Ead., Per la tradizione manoscritta, pp. 949-950.

14 Un'ipotesi analoga, sebbene non argomentata, è stata proposta da De Robertis, Scritture di libri scritture di notai, pp. 1-27, in partic. p. 23. 
nelle maiuscole al tratto del testo italiano (es. c. 8, rr. 9 e 13); un aspetto, questo, per nulla privo di significato se si considera che in tal caso non può trovarsi in fine di parola per un motivo semplicemente legato alla lingua utilizzata, il volgare per l'appunto. Inoltre, la fusione delle curve convesse tra le lettere $c$ ed $o$, che le rende simili ad un 8 rovesciato, messa in rilievo da Supino Martini per supportare l'analogia grafica tra testo volgare e commento, è presente altresì nella translatio latina (es. c. 69r; Fig. 11). Per avvalorare l'ipotesi dell'identità di mano, a questo livello di analisi vorrei affiancare un altro elemento che riguarda il sistema grafico di appartenenza delle scritture. Si può, dunque, concordare con Paola Supino Martini nel ritenere la scrittura del testo italiano e quella delle glosse appartenente al tessuto grafico della corsiva cancelleresca, piuttosto che a quello della littera textualis: ${ }^{15}$ lo evidenzierebbero, tra gli altri, l'aspetto arioso, il tracciato sottile, i legamenti tra le lettere, lo slancio delle aste alte spesso ad uncino (si veda la $d$ ), la terminazione ricurva al di sotto del rigo dei tratti bassi della $h$, in particolare, ma anche della $m$ e della $n$, la $a$ di forma corsiva, la $g$ 'a nove' e in legamento dall'alto, la $r$ in due tratti divaricati, che talvolta scende al di sotto del rigo, a cui aggiungerei anche l'utilizzo delle peculiari forme di $B$ e di $R$ maiuscole. Proseguendo su questa strada, e sulla scorta della tesi dell'identità di mano, anche la scrittura della traduzione latina può essere ricondotta al sostrato grafico cancelleresco, sebbene presenti, rispetto alle altre due grafie, un tracciato nel complesso più contrastato e spezzato, e maggiori elementi di contaminazione dalla coeva textualis, come, tra gli altri, la g per lo più testuale, e la $s$ tonda in fine di parola (es. c. 16r). In realtà, si tratta di elementi connotanti in senso demarcativo la littera textualis, che denotano piuttosto l'uso consapevole del copista di adottare - e quindi adattare ad una nuova funzione - una scrittura appartenente ad un ambito differente rispetto alla propria grafia usuale: anche qui ritroviamo, infatti, i tratti ricurvi al termine delle aste basse (si osservi soprattutto la $h$ ), lo slancio delle aste (tra cui la $d$ uncinata), la a corsiva, e all'opposto l'incostanza nella fusione delle curve contrapposte, come ad esempio nella $r$ tonda dopo curva convessa a destra, che spesso è diritta. In aggiunta, pare opportuno sottolineare come in alcune carte la grafia della translatio in latino diventi del tutto simile a quella utilizzata nei versi in volgare poiché il modulo si fa più piccolo, il tratteggio più sottile, e si accentua lo slancio delle aste (c. 69r; Fig. 11).

Dunque, della complessa progettualità dei Documenti d'Amore, evidenziata già ad un primo impatto visivo dalla mise en page a corone concentriche, fa parte integrante anche la scrittura che concorre a mettere in risalto l'ordine e la

15 Supino Martini, Per la tradizione manoscritta, pp. 949-950; Ead., Per la storia della semigotica, pp. 253-254; De Robertis, Scritture di libri scritture di notai. 
gerarchia dei tre livelli testuali, e quindi la «progressione culturale e sapienziale» ${ }^{16}$ del messaggio da essi veicolato, allo stesso modo degli altri espedienti utilizzati per esprimere simbolicamente i rapporti contenutistici e linguistici dell'insieme. ${ }^{17}$ D'altronde, l'assegnazione di un ruolo simbolico e culturale affidato alla scrittura, e la consapevolezza del rapporto intrinseco che la lega al testo, è tipico di tanti letterati dell'epoca (basti pensare a Francesco Petrarca), che riconoscevano a questa una funzione attiva e fondamentale nel processo di creazione e trasmissione del pensiero. ${ }^{18}$

\subsection{Il confronto grafico tra i due testimoni vaticani}

La questione dell'identificazione della mano che ha vergato il Barb. lat. 4076 può essere avvalorata, altresì, dal confronto con il secondo importante testimone dei Documenti d'Amore, il Barb. lat. 4077, esemplare provvisorio e incompleto dell'opera, latore del testo italiano (sebbene con lacune dovute alla perdita del secondo fascicolo), della traduzione latina alle carte $1 \mathrm{r}-5 \mathrm{r}$, e della glossa in latino unicamente sul recto della carta incipitaria (c. 1r; Fig. 15). L'incompiutezza contenutistica si riflette, tra l'altro, anche sul piano iconico, risultando qui mancante gran parte dell'apparato decorativo relativo al commento (delle 27 illustrazioni previste ne sono infatti presenti solo 18 , tracciate a penna e ad acquarello). Nonostante si rilevino differenze di stile nelle miniature, i due codici mostrano analogie nel formato, nell'impaginato, e nell'interazione tra componente testuale ed illustrativa. Per tale motivo è ancora oggi difficile stabilire l'anteriorità di un esemplare rispetto all'altro, riuscire a valutare quale dei due abbia funto da modello o, ancora (ipotesi che mi sembra più che plausibile), se entrambi si siano serviti a loro

16 Goldin Folena, Il commento di Francesco da Barberino, pp. 263-282; cit. p. 277; per lo stesso argomento cfr. ancora Goldin, Autotraduzione latina nei 'Documenti d'Amore', pp. 371-392; ed Ead., Testo e immagine.

17 Non a caso, a mio parere, la traduzione in latino viene in qualche modo "nobilitata" mediante l'utilizzo di una scrittura più aderente ai canoni della textualis - scrittura libraria per eccellenza -, che palesa il riconoscimento alla lingua latina di uno status ritenuto ancora ad un gradino culturale superiore rispetto al sermo vulgaris. D'altra parte, per controbilanciare la centralità conferita al testo in volgare, anche per la mise en page Barberino rimane ancorato alla tradizione gotica, scegliendo una impostazione del tutto in linea con i manoscritti universitari.

18 Petrucci, La scrittura di Francesco Petrarca, p. 62. Vedi anche de La Mare, The Handwriting of Italian Humanists, I.1, pp. 1-16; Petrucci, Libro e scrittura di Francesco Petrarca, pp. 5-20, in partic. p. 11. Vedi anche Signorini, La scrittura libraria di Francesco Petrarca, pp. 839-862; Battaglia Ricci, Edizioni d'autore, copie di lavoro, interventi di autoesegesi, pp. 123-57; Cursi, La scrittura e i libri del Boccaccio, pp. 83-128; e, da ultimo, Id., Scrivere, incidere, digitare, pp. 153 sgg. 
volta di un terzo codice di riferimento materialmente identico; ed infine le ragioni che si celano dietro all'interruzione della copia del Barb. lat. 4077. Per rispondere a tali interrogativi è necessario innanzitutto indagare alcuni aspetti legati al riconoscimento delle mani che intervengono nel Barb. lat. 4077. Anche in questo caso, la nuova analisi paleografica mi ha portato a rivalutare le teorie esposte finora, non solo relativamente al numero dei copisti e alla sequenza in base alla quale essi si succedono all'interno del manoscritto, ma altresì riguardo alle relazioni grafiche esistenti tra i due testimoni. Ma veniamo al primo punto. Il codice è trascritto da più mani, semigotiche e cancelleresche piuttosto minute, la prima delle quali (mano A), secondo una tesi avanzata inizialmente da Egidi, interviene nelle prime otto carte (vale a dire, nel testo in volgare delle cc. 1r-8r, nella traduzione latina delle cc. 1r-5r, e nella glossa di c. 1r; (cc. 1r, 4r; Figg. 15 e 16), nelle carte che ospitano le miniature, e in quelle ad esse affrontate (ovvero alle cc. 22v23r; 45v-46r; 49v-50r; 52v-53r; 54v-55r; 58v-59r; 73v-74r; 76v-79r; 81v-82r; 84v-85r; 87v-98r; es. c. 53r; Fig. 19); a questa mano, che lo studioso assimila alla mano A, ritenuta artefice unica del Barb. lat. 4076, egli affianca altri due scribi (mani B e C) nel testo italiano (cfr. Tab. 3). Armando Petrucci, dal canto suo, avvalorando in parte il pensiero di Egidi individua altre due amanuensi nelle prime otto carte del codice: uno (B) responsabile della traduzione latina e della glossa sulla carta incipitaria, e l'altro (C) della translatio in latino delle cc. 1v-5r, ribadendo, ad ogni modo, l'analogia grafica tra la mano A e quella che per Petrucci ha vergato il solo testo in volgare, più le cc. 99v-101v, nel Barb. lat. 4076 (cfr. Tab. 3); per entrambi gli studiosi essa è da identificarsi con quella di Francesco da Barberino. Pur concordando con la successione delle mani individuata da Egidi (e quindi con l'identificazione di un unico scriba nelle prime otto carte del codice), mi sembra tuttavia di poter riscontrare l'intervento di altri due copisti nelle carte trascritte dalla mano che lo studioso indica come C, e corrispondente alla mano E di Petrucci, che scrive in una gotichetta minuta, irregolare nell'andamento e nel tracciato, e con aste scarsamente slanciate; il primo amanuense è responsabile delle cc. 11r-14r, e utilizza una semigotica minuta e appena contrastata, con moderato slancio delle aste alte e con trattini di completamento uncinati per le basse; il secondo copia invece le cc. 14r-22r e 23v-45r, e adopera una semigotica di piccolo modulo, ben spaziata, slanciata, e con lettere piuttosto serrate tra loro; vedi Tab. 3a.

La suddetta analisi del Barb. lat. 4077, dunque, non solo rende possibile l'individuazione di cinque copisti e un loro diverso avvicendamento nel lavoro di trascrizione, ma permette anche di aggiungere elementi significativi alla tesi secondo cui il testimone completo dell'opera, il Barb. lat. 4076, sia stato vergato interamente da un unico scriba (a mio parere da identificarsi con l'autore stesso), e corrispondente alla mano A del Barb. lat. 4077. Tra gli aspetti grafici coincidenti si segnala la presenza nei versi in volgare dell'esemplare incompleto, oltre che della 
Tabella 3: Individuazione delle mani intervenute nel Barb. lat. 4077.

\begin{tabular}{|c|c|}
\hline Egidi (3 mani) & Petrucci (5 mani) \\
\hline $\begin{array}{l}\text { mano } A=\text { mano del Barb. lat. } 4076 \text { nella sua } \\
\text { interezza } \\
\text { - nelle cc. } 1 \mathrm{r}-8 \mathrm{r} \text { (testo italiano, latino, glossa) } \\
\text { - nel testo italiano delle carte con miniature e } \\
\text { in quelle ad esse affrontate }(22 \mathrm{v}-23 \mathrm{r} ; 45 \mathrm{v}-46 \mathrm{r} \text {; } \\
49 \mathrm{v}-50 \mathrm{r} ; 52 \mathrm{v}-53 \mathrm{r} ; 54 \mathrm{v}-55 \mathrm{r} ; 58 \mathrm{v}-59 \mathrm{r} ; 73 \mathrm{v}-74 \mathrm{r} ; \\
76 \mathrm{v}-79 \mathrm{r} ; 81 \mathrm{v}-82 \mathrm{r} ; 84 \mathrm{v}-85 \mathrm{r} ; 87 \mathrm{v}-98 \mathrm{r})\end{array}$ & $\begin{array}{l}\text { mano } A \text { = mano del solo testo volgare del } \\
\text { Barb. lat. } 4076 \\
\text { - nelle cc. } 1 \mathrm{r}-8 \mathrm{r} \text { (solo nel testo italiano) } \\
\text { - nel testo italiano delle carte con miniature e } \\
\text { in quelle ad esse affrontate }(22 \mathrm{v}-23 \mathrm{r} ; 45 \mathrm{v}-46 \mathrm{r} \text {; } \\
49 \mathrm{v}-50 \mathrm{r} ; 52 \mathrm{v}-53 \mathrm{r} ; 54 \mathrm{v}-55 \mathrm{r} ; 58 \mathrm{v}-59 \mathrm{r} ; 73 \mathrm{v}-74 \mathrm{r} ; \\
76 \mathrm{v}-79 \mathrm{r} ; 81 \mathrm{v}-82 \mathrm{r} ; 84 \mathrm{v}-85 \mathrm{r} ; 87 \mathrm{v}-98 \mathrm{r}) \\
\text { mano } B \\
\text { - a c. } 1 \mathrm{r} \text { (nella traslatio latina e nella glossa) } \\
\text { mano C } \\
\text { - nelle cc. } 1 \mathrm{v}-5 \mathrm{r} \text { (translatio latina) }\end{array}$ \\
\hline mano $B$ & mano $D$ \\
\hline $\begin{array}{l}\text { - nel testo italiano delle cc. } 8 \mathrm{v}-10 \mathrm{v} \\
\text { mano } C \\
\text { - nel testo italiano delle restanti carte del ms. } \\
\text { alternandosi alla mano } A(11 \mathrm{r}-22 \mathrm{r} ; 23 \mathrm{v}-45 \mathrm{r} ; \\
46 \mathrm{v}-49 \mathrm{r} ; 50 \mathrm{v}-52 \mathrm{r} ; 53 \mathrm{v}-54 \mathrm{r} ; 55 \mathrm{v}-58 \mathrm{r} ; 59 \mathrm{v}-73 \mathrm{r} ; \\
74 \mathrm{v}-76 \mathrm{r} ; 79 \mathrm{v}-81 \mathrm{r} ; 82 \mathrm{v}-84 \mathrm{r} ; 85 \mathrm{v}-87 \mathrm{r})\end{array}$ & $\begin{array}{l}\text { - nel testo italiano delle cc. } 8 \mathrm{v}-10 \mathrm{v} \\
\text { mano } E \\
\text { - nel testo italiano delle restanti carte del ms. } \\
\text { alternandosi alla mano } A(11 \mathrm{r}-22 \mathrm{r} ; 23 \mathrm{v}-45 \mathrm{r} ; \\
46 \mathrm{v}-49 \mathrm{r} ; 50 \mathrm{v}-52 \mathrm{r} ; 53 \mathrm{v}-54 \mathrm{r} ; 55 \mathrm{v}-58 \mathrm{r} ; 59 \mathrm{v}-73 \mathrm{r} ; \\
74 \mathrm{v}-76 \mathrm{r} ; 79 \mathrm{v}-81 \mathrm{r} ; 82 \mathrm{v}-84 \mathrm{r} ; 85 \mathrm{v}-87 \mathrm{r})\end{array}$ \\
\hline
\end{tabular}

Tabella 3a: Individuazione delle mani intervenute nel Barb. lat. 4077.

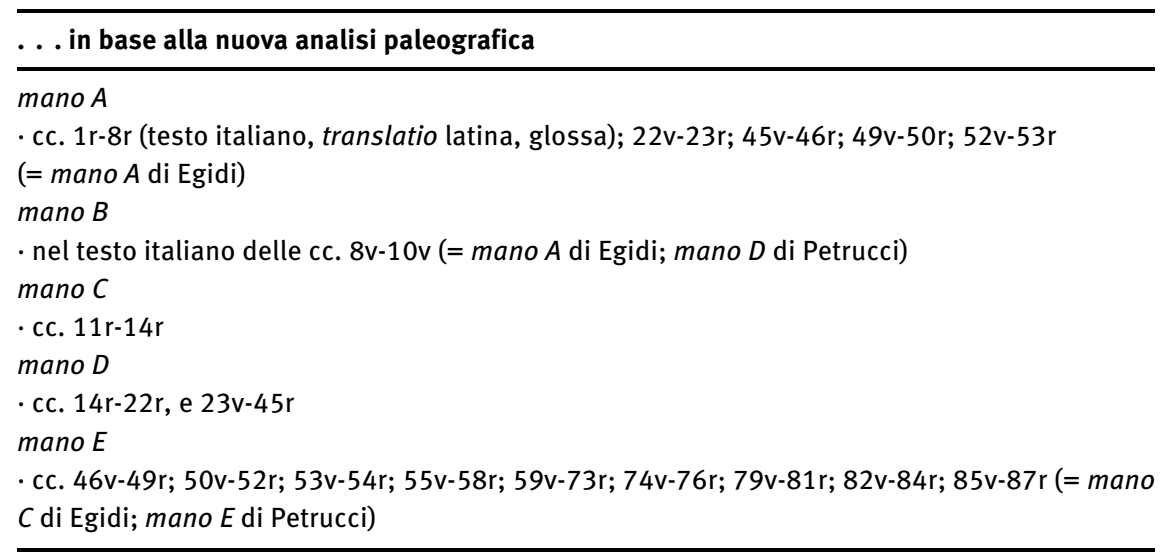

$g$ con occhiello inferiore ovale e spesso aperto - utilizzato pressoché esclusivamente nel codice precedente - di una $g$ di tipo testuale, che ricorda nella forma una $s$ maiuscola tagliata da un tratto trasversale (es. c. 5 r), che viene adoperata con maggior frequenza nella traduzione latina di entrambi i manoscritti. L'identità della mano A del Barb. lat 4077 con quella del Barb. lat. 4076, che è stata attri- 
buita, seppure in modo soltanto presunto, a Francesco, potrebbe inoltre contribuire a chiarire alcuni aspetti legati ai rapporti esistenti tra i due codici, dalle possibili motivazioni alla base dell'incompiutezza del primo, alle modalità di allestimento messe in atto dall'autore, anche riguardo all'apparato decorativo. Non entrando nel merito dell'autografia relativa alle illustrazioni, l'esame paleografico potrebbe in effetti fornire qualche apporto in più, analogamente ad altri elementi extra-testuali, per proporre anzitutto valutazioni su tempi e modi di realizzazione delle miniature. Ad esempio, il fatto che la scrittura di tutte le carte miniate del 4077, e di quelle a fronte, siano della stessa mano, suggerisce una anteriorità nell'esecuzione della componente iconica rispetto a quella testuale (suggestione, tra l'altro, già proposta da Supino Martini anche per il 4076), ${ }^{19}$ secondo una modalità di allestimento che dobbiamo immaginarci immediatamente consequenziale, finalizzata a mantenere la specularità dell'impaginato ad apertura di libro, ragione per la quale, contrariamente alla prassi, si eseguirono prima le immagini, quindi il testo della pagina relativa, e quello del recto o verso della carta affiancata.

L'incompiutezza del codice (sia a livello contenutistico che decorativo) costituirebbe, tra l'altro, un ulteriore indizio utile a ricostruire la sequenza della stesura dei tre differenti blocchi testuali: si può ragionevolmente pensare ad una iniziale trascrizione del testo italiano (che è presente nella sua interezza), seguito da quello latino (interrotto alla quinta carta), e per ultimo da quello della glossa (trascritto unicamente sulla carta incipitaria) - non a caso le miniature incompiute sono proprio quelle che, nell'idea originaria, dovevano accompagnare il commento. A rendere ancora più plausibile l'antecedenza dell'apparato illustrativo rispetto a quello testuale concorre anche la presenza costante (cioè anche laddove manca il testo relativo) delle iniziali miniate per la traduzione latina, e di quelle, tuttavia solo abbozzate, delle glosse, in corrispondenza dell'incipit di ogni Documento. $^{20}$ Inoltre, se si osservano le glosse del Barb. lat. 4076 accanto alle scene figurate queste risultano variabili, ovvero si adattano alla miniatura seguendo la forma dei riquadri illustrativi (c. 64r; Fig. 10), ${ }^{21}$ a riprova ulteriore di una quasi certa precedenza nella realizzazione dell'apparato iconico rispetto a quello verbale, che rivela altresì una analoga metodologia di lavoro per entrambi

19 Supino Martini, Per la tradizione manoscritta, p. 949.

20 Testimonierebbero ciò anche alcune rubriche e didascalie che appaiono chiare indicazioni per il miniatore (vedi c. 49v: «hoc ad glosas pertinet non ad testum», e c. 87v: «hoc spatium ad glosas pertinet non ad testum»). A questo proposito vedi Supino Martini, Per la tradizione manoscritta, p. 946; Panzera, Per l'edizione critica, p. 99.

21 Si vedano, a mo' di esempio, le cc. 32v, 64r; sulla c. 64r, inoltre, anche il titolo corrente relativo alla parte in cui si suddividono i Documenti è trascritto al di sopra del riquadro miniato. 
i codici. Alla base dell'anteriorità della componente figurativa potrebbero allora intravedersi non soltanto fattori di ordine funzionale, ma anche, e forse soprattutto, motivazioni di carattere simbolico correlate alla finalità stessa delle immagini, che assumono la valenza di veri e propri corredi interpretativi - potremmo dire di veicoli "significanti" -, e sembrano quindi nascere nella mente dell'autore ancor prima del testo stesso. ${ }^{22}$ Riguardo, invece, al rapporto temporale tra i due esemplari, si può concordare con le tesi avanzate sinora relativamente alla precedenza del Barb. lat. 4077 rispetto al codice completo, sulla base di elementi di natura extra-testuale (come quelli stilistico-figurativi), ma anche di natura interna, come, ad esempio, quelli desunti dallo studio delle varianti, che è stato approfondito in questa stessa sede da Cristiano Lorenzi e Antonio Montefusco, mediante l'analisi delle correzioni e delle riscritture presenti tanto nella traduzione latina quanto nelle glosse (meno nel testo volgare) del Barb. lat. $4076 .^{23}$ Sulla base di ciò, si potrebbe supporre che il progetto iniziale dapprima applicato al 4077, ove già si scorge l'idea di realizzare un impaginato strutturato su più livelli

22 Cfr. Goldin, Testo e immagine, pp. 133-135. Per la questione relativa all'accezione di immagine verbale vedi Pozzi, Dall'orlo del visibile parlare, pp. 15-41.

23 Per quel che riguarda la datazione del Barb. lat. 4077 sulla scorta di elementi extra-testuali vedi per primo Egidi, Le miniature dei codici Barberiniani, pp. 2-3; Francesco da Barberino, I Documenti d'Amore, IV, pp. XXXV-XXXVII. Lo studioso ipotizza che l'allestimento del manoscritto, una delle prime copie dell'opera, sia avvenuta intorno al 1308, ovvero quando il Barberino era in esilio in Italia, e che sia stato poi concluso durante il suo soggiorno in Provenza (1309-1313); qui sarebbe poi cominciata la stesura del testimone completo, terminata con ogni probabilità al rientro dell'autore in Italia, sicuramente in un momento non posteriore al 1313, anno di morte dell'imperatore Enrico VII che invece viene considerato ancora vivente in un passo a c. $94 \mathrm{r}$ del Barb. lat. 4076. Per la precedenza temporale del 4077 vedi anche Petrucci, Minuta, autografo, libro d'autore, pp. 409-410; Id., Minima barberina, p. 1007; Panzera, Per l'edizione critica, pp. 98-99. Riguardo all'importanza delle varianti e del complesso procedimento di revisione testuale per ipotizzare una data di composizione del manufatto cfr. in particolare Supino Martini, Per la tradizione manoscritta, pp. 951-953. Per le motivazioni di carattere stilistico-decorativo che accosterebbero il Barb. lat. 4077 agli esempi iconografici padovani, e che permetterebbero di collocarlo in un momento ancora precedente rispetto a quanto supposto da Egidi, ovvero tra il 1304 e il 1308, cfr. Ciccuto, Francesco da Barberino e il suo Bildercodex, p. 88. Un'origine veneziana o padovana viene avanzata anche da Boskovits, The Fourteenth Century, p. 191, n. 13, che ascrive alla mano di Francesco anche il disegno abbozzato del Trionfo d'Amore nel noto Canzoniere di Niccolò de Rossi (Città del Vaticano, Biblioteca Apostolica Vaticana, Barb. lat. 3953), per cui si veda da ultimo la scheda curata da Maddalena Signorini, con riferimenti bibliografici aggiornati, per la mostra «Dante e il suo tempo nelle biblioteche fiorentine», in programma dal 23 settembre 2021 al 15 gennaio 2022. un influsso sul Barberino del Giotto padovano si legge anche in Nardi, Le illustrazioni dei «Documenti d'Amore», pp. 75-92. Per gli aspetti di natura filologica e testuale che consentono di avanzare l'ipotesi dell'anteriorità del codice incompleto sul Barb. lat. 4076 vedi in questo stesso volume i contributi di Lorenzi e Montefusco rispettivamente alle pp. 37-49 e alle pp. 51-63. 
testuali (comprensivo anche della glossa), oltre a quello iconico, venne poi abbandonato per ragioni probabilmente legate ad un'insoddisfazione per l'estetica e per la scorrettezza testuale, e riproposto nel 4076 variando qualche elemento (decorativo e contenutistico), ma pur sempre ripresentando il medesimo modello progettuale, poiché del tutto simili appaiono - come si diceva - la mise en page, il formato, e le interazioni tra le componenti testuali e decorative dei due esemplari. Il Barb. lat. 4077 costituirebbe così un originale provvisorio (ma, presumo, non nelle intenzioni iniziali del suo autore) trasformatosi poi in minuta, e il Barb. lat. 4076 il testimone completo e definitivo, a mio parere derivato a sua volta da un ulteriore antigrafo di riferimento, che ha funto da modello per entrambi. ${ }^{24}$ Del resto se si analizzano i cospicui interventi correttivi marginali e interlineari presenti nel codice completo, avvenuti su rasura o mediante riscrittura, e attribuiti anch'essi alla mano di Francesco, non si fa fatica a notare un attento lavoro di revisione autoriale, visibile in particolar modo nel commento latino, e che potrebbe anche avviare, a mio parere, una discussione legata all'accezione stessa da conferire al termine "definitivo". Si potrebbe infatti pensare che il Barb. lat. 4076 fosse sì progettato per diventare un'edizione definitiva dell'opera, ma con il tempo fosse poi divenuto un esemplare "da lavoro", seppure di fattura insigne, rimasto sullo scrittoio del suo autore insieme al 4077 (e, forse, insieme ad altre minute), il quale fu terminato solo in un momento successivo, da inabili amanuensi, presumibilmente quando Francesco era già morto, come lascerebbero intendere l'abbandono della struttura a "corone concentriche", e il completamento della sola parte in volgare. ${ }^{25}$ Tale aspetto non stupisce se si considera che la tradizione manoscritta dei Documenti d'Amore, limitata soltanto ad altri quattro esemplari (a cui si devono aggiungere due testimoni seicenteschi che tentano una ricostruzione del commento), è legata al solo testo italiano, persino in un codice prossimo all'allestimento del Barb. lat. 4076, ovvero il Barb. lat. 4028, probabilmente approntato quando l'autore era ancora in vita (cioè prima del 1348), e l'unico a mostrare una mise en page del tutto identica a quella dei due codici più noti. ${ }^{26}$ Le ragioni celate

24 Lo stesso autore ci informa che l'opera fu redatta in almeno quattro versioni; a c. $24 \mathrm{v}$ del Barb. lat. 4076 in un passo della glossa si legge, infatti: «non sit lictera in hoc liber nec figura que, ante alicuius transcriptum, per me ad minus non fuerit tracta quater».

25 Lo stato di "non finito" del Barb. lat. 4076 è palesato anche dalle iniziali di paragrafo che, seppure di modulo maggiore e a doppia filettatura, rimangono allo stato di "bozze", poiché tracciate nello stesso inchiostro del testo, analogamente ai titoli correnti. La modifica della natura del codice che, mediante un'attenta attività di correzione e revisione, finisce per perdere un po' la sua aurea di esemplare definitivo e di lusso era già stata messa in luce da Armando Petrucci in Petrucci, Minuta, autografo, libro d'autore, p. 410.

26 I codici latori dell'opera sono: Città del Vaticano, Biblioteca Apostolica Vaticana, Barb. lat. 4028 (ante 1348); Firenze, Biblioteca Nazionale Centrale, Banco Rari 72 (sec. XIV), Firenze, Bi- 
dietro al successivo abbandono del complesso impianto progettuale di autotraduzione con commento marginale andrebbero probabilmente ricercate sia nell'interesse specifico di tramandare il solo testo in volgare (perché, forse, comprensibile ai più?), sia nella conseguente mancata ricezione dell'idea originaria dell'autore, e dunque della sua portata innovativa, che inevitabilmente condusse al fallimento di un'impresa editoriale ancora anacronistica e inattuabile per l'epoca, e che non riuscì a creare una tradizione a sé stante. ${ }^{27}$

\subsection{La questione dell'autografia}

Quanto argomentato sinora ci introduce inevitabilmente all'interno della delicata questione dell'autografia (a cui si legano le accezioni stesse di autenticità e autorialità dell'impresa) fino a questo momento solo accennata, e di cui vorrei brevemente discutere prima di avviarmi alla conclusione, per formulare alcune considerazioni che mi auguro possano essere almeno dei suggerimenti per approfondimenti futuri. Come già discusso in precedenza, l'autografia totale del Barb. lat. 4076 era stata sostenuta da Egidi che riconosceva, tra l'altro, la stessa mano anche nelle prime otto carte del Barb. lat. 4077, in quelle con le miniature,

blioteca Riccardiana, ms. 1060 (secc. XIV-XV), e Firenze, Biblioteca Nazionale Centrale, Magliab. VIII 683 (del XV secolo); e il seicentesco: Città del Vaticano, Biblioteca Apostolica Vaticana, Chigiano L.V.169.

27 Nonostante la complessità del progetto barberiniano, l'autore scelse comunque un modello librario inserito nel solco della tradizione, optando per una impaginazione complessa, ma che ricalcava la tipologia del libro universitario. Di contro, le soluzioni adottate da Petrarca per i suoi autografi, seppure protesero verso un modello anti-universitario, conobbero una ricezione migliore rispetto a quelli del Barberino: un esempio è il Canzoniere, che riuscì a creare una seppur minima tradizione, il che sottenderebbe una certa comprensione del significato insito nel modello-libro proposto dall'autore; per tale argomento cfr. Signorini, Fortuna del "modellolibro" Canzoniere, pp. 133-154, e Cursi, Per la prima circolazione dei Rerum vulgarium fragmenta, pp. 225-261; vedi anche Petrucci, Spazi dei testi e strategie petrarchesche, pp. 45-56, in partic. p. 52. Sulla portata innovativa del Petrarca "editore", e su quella del suo contemporaneo Boccaccio, vedi Battaglia Ricci, Edizioni d'autore, copie di lavoro, autogenesi; per le scelte di determinate forme-libro, messe in atto dal Boccaccio in base alle tipologie testuali, cfr. in particolare Cursi, Decameron; Id., La scrittura e i libri di Giovanni Boccaccio, pp. 83-128; e anche Cursi, Boccaccio architetto e artefice di libri, pp. 35-62. Come per Petrarca, anche per il Decameron boccacciano esiste una tradizione, ristretta a soli cinque manoscritti (tre dei quali appartenenti alla c.d. "proto-diffusione"), che sembra evidenziare l'aderenza verso la concezione di una specifica forma libraria proposta dall'autore, che finisce per assumere il ruolo di modello caratterizzante (cfr., di nuovo, Cursi, Decameron; Id., La scrittura e i libri di Giovanni Boccaccio, pp. 107-128). 
e nelle pagine ad esse contigue. ${ }^{28}$ Per Petrucci, invece, l'intervento autografo nel codice 4076 era limitato al testo poetico in volgare, alle cc. 99v-101v, nonché alle correzioni in margine e in interlinea, vergate in una scrittura più minuta e corsiveggiante. Nel manoscritto "provvisorio" lo scriba principale era invece riconoscibile nel solo testo in volgare delle prime otto carte, nelle pagine ospitanti le illustrazioni, e in quelle a fronte, ma anche nelle didascalie e nelle istruzioni per il miniatore, come suggerito dall'attinenza grafica con le correzioni del Barb. lat. 4076. Tanto Egidi quanto Petrucci basavano le loro ipotesi su elementi interni al testo poiché per entrambi il raffronto con i documenti notarili autografi era risultato improduttivo, vista l'adozione in essi di una corsiva cancelleresca formalizzata, del tutto differente tipologicamente dalla scrittura utilizzata nei codici. ${ }^{29}$ Distante dalle posizioni di Egidi, secondo il quale le affermazioni presenti nelle glosse relative alle sospensioni del lavoro di trascrizione costituivano prove dirette dell'autografia dell'autore, ${ }^{30}$ Petrucci riconosceva a Francesco piuttosto un'opera di riscrittura e di revisione autoriale a più livelli, e in più fasi di elaborazione del testo, dimostrata sia dagli interventi correttivi autografi, sia da alcune dichiarazioni esplicite presenti nel commento, attribuendo così la maggior parte della trascrizione a copisti di professione. ${ }^{31}$ Premesso ciò, sarei invece propensa a sostenere, come già accennato prima, che la mano a mio parere responsabile dell'intero Barb. lat. 4076 appartenga all'autore stesso, tanto più che anche gli interventi di revisione marginali e interlineari (già ritenuti autografi di Francesco) sono riconducibili ad essa, in linea con le affermazioni espresse dall'autore in alcuni punti del testo, relative ad una sua partecipazione attiva nel lavoro di trascrizione, effettuato mediante la stesura di redazioni intermedie prima di giungere a quella definitiva. ${ }^{32}$ Proprio tali asserzioni sono, a mio avviso, prove importanti

28 Francesco da Barberino, I Documenti d'Amore (ed. Egidi), pp. XX sgg.

29 Ibidem; Petrucci, Minima barberina, p. 1008; la stessa Cristina Panzera, dopo aver consultato tre documenti rogati o sottoscritti dal Barberino conservati all'Archivio di Stato di Firenze (Diplomatico, R. Acquisto Menozzi, 18 febbraio 1299; Cestello, 16 agosto 1300 e S. Maria Novella, 25 settembre 1299) afferma che: «La cancelleresca di Francesco è piccola e rotonda e genericamente accostabile alla mano del testo volgare» (Panzera, Per l'edizione critica, p. 96, n. 14).

30 Le numerose annotazioni presenti nel commento sono elencate in Francesco da Barberino, I Documenti d'Amore (ed. Egidi), IV, p. XXI, n. 1.

31 Vedi Petrucci, Minima barberina, p. 1009. Di analoga opinione anche Paola Supino Martini che attribuisce gran parte del Barb. lat. 4076 AD uno scriba di professione (Supino Martini, Per la tradizione manoscritta, pp. 953-954).

32 Un riferimento all'attività di revisione messa in atto da Francesco da Barberino si legge, ad esempio, alla c. $24 \mathrm{r}$ del Barb. lat. 4076: «corrigendo rescripsi et rescripta iterum et iterato correxi et hic porrigo pro correctis, ut est hominis in hoc posse» (cfr. Francesco da Barberino, $I$ Documenti d'Amore (ed. Egidi), IV, p. XXII). 
non solo per convalidarne l'autografia, ma anche per appurare le modalità compositive adottate dall'autore nell'elaborazione e nella messa per iscritto delle sue opere, che evidentemente procedevano per stadi provvisori finalizzati al raggiungimento della copia in pulito, atta alla "pubblicazione". 33 Se, dunque, come credo, si può avvalorare l'ipotesi di autografia autoriale per il manoscritto 4076, ci troveremmo davanti alla prima opera letteraria appartenente alla tradizione italiana nella quale auctor e scriptor coincidono, e dunque davanti al primo caso di «autografia d'autore», dove l'azione di auto-scrittura si fonda con quella di riscrittura, secondo una prassi ben nota nel mondo notarile, ed ora trasferita nel contesto librario. ${ }^{34} \mathrm{E}$ qui in nuce quindi quello che possiamo già definire "libro d'autore" $\mathrm{o}$, meglio ancora, «edizione d'autore autografa», ${ }^{35}$ secondo un processo che condurrà prima con Petrarca, poi con Boccaccio, e successivamente con Coluccio Salutati e con tutta una generazione di umanisti, al superamento del modello notarile e gra-

33 D'altra parte, analoghe modalità di composizione sembrano caratterizzare anche il Reggimento e costumi di donna, così come ci informa lo stesso Francesco in un passo della glossa dei Documenti d'Amore, a c. 4v del Barb. lat. 4076, dove l'autore fa riferimento ad una necessaria operazione di rescriptionem et expeditionesm totalem di cui abbisognava l'opera, lasciata in quel momento in sospeso per la mancata disponibilità dei quaternos interlineatos. Interessante notare, a mio parere, anche la menzione che il Barberino fa di tali quaternos, da intendersi verosimilmente con fascicoli già opportunamente rigati per ospitare la scrittura; siffatta allusione ci fornisce infatti informazioni dirette sui metodi di copia seguiti all'epoca dagli amanuensi, i quali erano soliti trascrivere su fascicoli sciolti che solo in un secondo momento venivano rilegati. Per la trascrizione completa del passo in questione cfr. Francesco da Barberino, I Documenti d'Amore (ed. Egidi), I, pp. 33-34; per il suo commento cfr. Battaglia Ricci, Comporre il testo, p. 26; vedi, da ultimo, Giunti, Sulla struttura formale del Reggimento, p. 95, ove si analizza nello specifico la struttura del manoscritto conservato presso la Biblioteca Apostolica Vaticana, il Barb. lat. 4001, testimone principale dell'opera, e con ogni probabilità apografo diretto dell'originale.

34 Per tale argomento cfr., in particolare, Petrucci, La scrittura del testo; Id., Dalla minuta al libro d'autore, pp. 364 sgg.; ma anche Bologna, Tradizione e fortuna dei classici italiani, p. 145. Per una panoramica più ampia del concetto di autografia vedi Bartoli Langeli, Autografia e paleografia, pp. 41-60.

35 Ancor più che di «libro d'autore», nel caso di Francesco da Barberino (come per Petrarca e Boccaccio) si può parlare, a mio avviso, di «edizione d'autore», in ragione del fatto che l'autore ha in mente e allestisce una specifica forma-libro, comprensiva anche di elementi para-testuali funzionali ad una sua pubblicazione, almeno intenzionale; per un approfondimento su tale tematica cfr. Battaglia Ricci, Edizioni d'autore, copie di lavoro, autogenesi, p. 135. Una definizione di edizione per i manoscritti del Canzoniere petrarchesco è presente anche in Feo, Francesco Petrarca, pp. 277-278: «Alla prima seguono altre edizioni del libro delle rime, intendendo Petrarca per edizione quello che aveva inteso Cassiodoro, ossia la sistemazione di un codex archetypus, di un testo approvato dal suo autore e da lui mandato ad almeno una persona con facoltà di trarne altre copie»; vedi anche Zamponi, Il libro del Canzoniere. 
zie al progressivo delinearsi di una vera e propria figura di letterato, che revisiona in prima persona e sottopone a riformulazioni letterarie continue il proprio lavoro, così che l'esemplare originale finisce spesso per assumere le caratteristiche di un «codice-archivio del testo», ${ }^{36}$ del quale è possibile rilevare progressivamente i vari e successivi stadi di elaborazione, tutti allo stesso modo autentici. ${ }^{37}$ In tal senso, Francesco sembra farsi precursore, ancor prima di Petrarca, di una serie di innovazioni di rilievo che riguardano in primo luogo l'organizzazione grafico-visiva del libro; questo assume quasi le sembianze di un vero progetto "editoriale" (o almeno così doveva essere nelle intenzioni dell'autore), pensato in ogni sua parte, e seguito in ogni sua fase, dalla bozza iniziale all'exemplar "definitivo", e che palesa la volontà del Barberino di contrapporsi al sistema di produzione librario vigente, meccanico e seriale (la stessa reticenza che sarà poi di Petrarca), nonché una netta protesta nei confronti dei copisti del suo tempo, accusati di essere ignoranti e incapaci. ${ }^{38}$ Alla natura emulativa insita nell'attività di copia l'autore contrappone infatti la superiorità di quella creativa, che presuppone una serie di accorgimenti e di scelte grafico-librarie (anche di carattere stilistico-decorative) tutt'altro che casuali, finalizzate a rendere il libro, nella sua materialità storica, un prodotto culturale destinato a circoscritti e specifici destinatari (siano essi reali o solamente immaginati). ${ }^{39} \mathrm{~A}$ questo aspetto è strettamente collegato anche quello ben più ampio che concerne l'interesse di Francesco nei riguardi del lento processo di attestazione del sermo vulgaris, ancora subordinato alla permanente funzione normativa della lingua latina, ma della cui portata innovativa l'autore si fa testimone consapevole attraverso l'opera di auto-traduzione dei Documenti d'Amore, dove la complementarità dei due registri linguistici viene espressa anche grazie

36 Vedi Petrucci, Minuta, autografo, libro d'autore, p. 403. Immediato il confronto con il noto Canzoniere vaticano, parzialmente autografo di Petrarca, il Vat. lat. 3195, per il quale cfr. Zamponi, Il libro del Canzoniere.

37 Petrucci, Modello notarile e testualità, p. 141; Id., Minuta, autografo, libro d'autore. Per il rapporto tra Autore-Testo-Lettore, in un'ampia panoramica d'insieme, cfr. Antonelli, Il testo fra Autore e Lettore.

38 Petrucci, Minuta, autografo, libro d'autore, pp. 409-410; Id., La scrittura del testo, pp. 289-290; Id., Scrivere il testo, 224-225; Id., Storia e geografia delle culture scritte, pp. 1226-1127. Per ciò che riguarda le scelte codicologiche e grafiche di Petrarca, e del "modello-libro" Canzoniere, cfr. Signorini, Fortuna del "modello-libro" Canzoniere, pp. 133-154.

39 Petrucci, La scrittura del testo, p. 138; Id., Scrivere il testo; Id. La scrittura del testo, pp. 289-291; Id., Dalla minuta al libro d'autore, p. 364 sgg. La superiorità creativa dell'artista rispetto all'attività di copia dello scriba è affermata da Francesco in un punto del commento dei Documenti, presente a c. 18r del Barb. lat. 4076: «maior subtilitas est in pinctore qui de iure suo subtilia format quam in scriptore qui scripta esemplando transummit», per cui cfr. Francesco da Barberino, I Documenti d'Amore (ed. Egidi), I, p. 94. 
all'ausilio di un preciso modello librario, avvertito dall'autore stesso «come tratto significante del sistema-testo». ${ }^{40}$ dunque mediante una gerarchia grafica visibile sia nella costruzione spaziale dell'impianto sia nell'adozione voluta di scritture contraddistinte, seppure unicamente negli aspetti sovrastrutturali e sub-liminali. ${ }^{41} \mathrm{La}$ scelta dell'autore di adattare all'ambito librario la propria grafia usuale mediante stilemi appartenenti alla littera textualis rivela una intenzionalità programmatica a priori finalizzata a collocare il manufatto entro una tradizione consolidata, che presuppone non solo il riconoscimento di specifiche funzioni assolte di volta in volta dalla scrittura, ma anche l'assegnazione di un valore ontologico all'atto dello scrivere che, in quanto tale, diviene lo strumento principale per veicolare il messaggio del testo. ${ }^{42}$ Alla scrittura, infatti, si conferisce anche il ruolo di mettere in relazione "semantica" i due registri linguistici, poiché se da un lato la scelta di un analogo sistema grafico pone sullo stesso piano il volgare e il latino, dall'altro l'aderenza più spiccata verso i caratteri tipici della textualis nella translatio cela, a mio avviso, una volontà seppur minima di "nobilitare" la lingua latina, alla quale si riconosce ancora una funzione esplicativo-esegetica nei confronti del volgare. ${ }^{43} \mathrm{La}$ peculiare percezione della potenza espressiva dei fatti grafici e dello stretto connubio esistente tra testo e libro, il valore riconosciuto all'autografia come unica garanzia per una perfetta (nonché perfettibile) testualità, grazie alla costante attività di

40 Battaglia Ricci, Boccaccio, p. 126. Sulle ragioni del bilinguismo barberiniano cfr. Goldin, Autotraduzione latina nei «Documenti d'Amore», pp. 371-392; Petrucci, Minuta, autografo, libro d'autore, p. 410.

41 L'utilizzo di scritture differenti a seconda degli usi e dei contesti grafici (librario e documentario) si colloca all'interno di quell'ampio fenomeno denominato "multigrafismo relativo" che accomuna tanti notai e letterati dell'epoca, per cui cfr. Petrucci, Funzione della scrittura e terminologia paleografica, I, pp. 1-30, in partic. pp. 22-23. Lo stesso fenomeno è stato indagato e approfondito in anni recenti da De Robertis, Digrafia nel Trecento, pp. 221-235; vedi anche Ead., Una mano tante scritture, pp. 18-38.

42 L'adozione consapevole di scritture appartenenti al sostrato grafico della littera textualis per l'ambito librario (non solo per i testi latini, ma anche per quelli in volgare) appare in linea di tendenza con le scelte di altri noti letterati dell'epoca, quali gli stessi Petrarca e Boccaccio, ma anche di notai-copisti del rango di Andrea Lancia, e denota la chiara distinzione che si faceva a questa altezza cronologica tra i genera scribenda, tanto da riceverne anche una teorizzazione nelle summae di ars prosandi, come quella di Conradus de Mure: «Alia enim manus requiritur in quaternis scribendis, et alia in epistolis»; cfr. Rockinger, Briefsteller und Formelbücher, p. 439.

43 D'altra parte, è l'autore stesso che in un punto del Reggimento e costumi di donna, parlando del Barb. lat. 4076 fa riferimento alla superiorità di coloro che conoscono il latino rispetto ai lettori monolingue: «ivi è uno testo volgar per la gente / ch'a più non è intendente, / e, intorno a quello, un testo letterale / per chi più sa vale» (Francesco da Barberino, Reggimento e costumi di donna (ed. Sansone), pp. 43-44; a tal proposito cfr. anche Goldin, Testo e immagine, p. 133). 
revisione che rende l'opera stessa (come il contenitore atto a tramandarla) un prodotto culturale continuamente in-fieri, e la struttura stessa dell'impaginato che sembra proporre uno specifico modello librario, sono tutti elementi che a mio avviso contribuiscono a rendere evidente la strategia compositiva dell'operazione messa in atto dal Barberino. Proprio in quest'ottica, e sulla base di quanto argomentato sino ad ora, diviene allora altamente probabile una responsabilità autoriale nell'attività di copia dei due esemplari "originali”. Se l'individuazione di una stessa mano nel Barb. lat. 4076, identificabile con uno degli amanuensi (mano A) intervenuti anche nel Barb. lat. 4077, è stata resa possibile grazie all'expertise paleografica, questa tuttavia non può assumere un'analoga efficacia nel confronto grafico tra i due testimoni e le pergamene notarili vergate da Francesco, poiché l'utilizzo di un'elegante e artificiosa minuscola cancelleresca per l'ambito documentario rende assai difficoltoso scegliere su quali parametri fondare l'analisi paleografica per far sì che la scrittura diventi di per sé "riconoscibile", e dunque comparabile. Nonostante l'esame dei documenti barberiniani sia stato da sempre considerato di scarso valore probante per ipotizzare l'autografia dei codici vaticani, mi pare che una prima indagine da me condotta sulle pergamene conservate nel fondo Diplomatico dell'Archivio di Stato di Firenze si sia invece rivelata di una qualche utilità. ${ }^{44}$ In particolare, la presenza di alcune lettere assimilabili morfologicamente alla scrittura dei due manoscritti mi sembra fatto di non poco conto, considerando anche che l'analisi è stata condotta procedendo per piani intrecciati, vale a dire comparando la grafia dei due esemplari non solo con quella adoperata nei documenti notarili di Francesco, ma anche con quella utilizzata, negli stessi documenti, dal poeta stilnovista Lapo Gianni, notaio e collaboratore assiduo del Barberino. ${ }^{45}$ Nonostante la sostanziale uniformità tra le due scritture, che denota una reciproca influenza grafica, a mio avviso è possibile riconoscere alcune caratteristiche peculiari nel modus scribendi di Francesco che lo accosterebbero alla mano dei due testimoni barberiniani; non solo nel tratteggio marcato, e allo stesso tempo fluido, della scrittura, come pure nell'elegante rotondità delle lettere (elementi questi che la contraddistinguono dalla sottile, e più angolosa, grafia di Lapo Gianni), ma anche e soprattutto nella morfologia di alcune lettere

44 Le pergamene da me analizzate sono state: ASFi, Diplomatico, Normali, Firenze, Sant'Ambrogio (Benedettine) 3, ottobre 1299; Diplomatico, R. Acquisto Menozzi, 18 febbraio 1299; S. Maria Novella, 25 settembre 1299. Per un elenco dei documenti cfr. Azzetta, Tra gli amici e $i$ cultori di Dante, pp. 61-71.

45 Per un elenco dei documenti vergati da Francesco da Barberino e da Lapo Gianni cfr. Azzetta, Tra gli amici e i cultori di Dante, pp. 61-71; per il rapporto grafico tra le scritture dei due notai, e più in generale per le analogie stilistiche che accomunano i collaboratori del Barberino vedi Ceccherini, Teaching, Function and Social Diffusion of Writing, pp. 177-192. 
come, ad esempio: la $r$ con i due tratti divaricati; la $s$ tonda a forma di 8 ; la $M$ maiuscola, tracciata in due tempi, con il primo tratto in foggia di a corsiva, e il secondo formato da un tratto ricurvo che discende al di sotto del rigo; la $N$ maiuscola di forma onciale e rotondeggiante; e il caratteristico legamento co simile ad un 8 rovesciato. Credo che un'indagine di tal genere necessiterebbe di ulteriori approfondimenti, non solo in termini quantitativi, ovvero mediante l'esame autoptico di tutte le pergamene vergate dal Barberino, ma anche e soprattutto in termini qualitativi, vale a dire prendendo in considerazione, oltre alla morfologia e al ductus, tutti quelli aspetti caratterizzanti che contribuiscono a ritenere distintiva e qualificante una data scrittura, e a renderla così comparabile - se ciò è davvero possibile - con le sue diverse "espressioni grafiche" (anche quelle appartenenti a contesti grafici differenti). In tal senso sarebbe quindi auspicabile valutare non solo gli elementi similari, ma anche quelli differenzianti, a cominciare dalle varianti di lettera (in particolar modo delle lettere diacritiche, come la $d$ e la $g$ ) e, soprattutto, ad analizzare i fatti grafici nel loro complesso che possono rilevare abitudini o automatismi messi in atto dai copisti, e che talvolta sfuggono al loro controllo. Tra questi la fusione delle curve contrapposte, l'elisione o la sovrapposizione dei tratti di attacco o di stacco, la chiusura di alcune lettere aperte sulle successive (ad esempio della $c$ ), o ancora la presenza di un'ideale catena grafica creata dai tratti di attacco e di stacco, oltre a tutti quegli elementi "accessori" che riguardano in sostanza lo stile e l'aspetto di una scrittura. ${ }^{46}$ Sulla base di ciò, mi sembra chiaro che l'indagine condotta fin qui sugli autografi notarili sia ancora ad una fase iniziale e indiziaria, ma forse utile per apportare qualche contributo ulteriore allo studio di una personalità grafica come quella di Francesco da Barberino che mostra di essere, rispetto ai suoi contemporanei, ancora più consapevole del rapporto intrinseco che intercorre tra l'oggetto-libro e il testo, come pure dei legami imprescindibili che si instaurano tra ogni componente strutturale del codice (in primo luogo tra la scrittura e le immagini), tanto che anche il manoscritto diviene, nella sua stessa materialità, un prodotto culturale capace di veicolare in maniera esplicita il messaggio implicito dell'autore. Ed è proprio in questo, a mio parere, la portata innovativa del Barberino che, quanto a sperimentazione di modelli grafico-librari e a presa di co-

46 Sull'importanza di un approccio paleografico di tal genere pone l'attenzione Teresa de Robertis per esaminare le scritture di quegli amanuensi che utilizzano in modo intercambiabile scritture appartenenti a sistemi grafici diversi, per cui cfr. De Robertis, Digrafia nel Trecento, p. 235. Su questa scorta vedi anche la metodologia messa in atto in Zamponi, Pantarotto, Tomiello, La stratigrafia dello Zibaldone, pp. 181-258, in particolare le pp. 206-226, per analizzare gli autografi boccacceschi della Biblioteca Laurenziana, vale a dire lo Zibaldone Plut. 29.8 e la Miscellanea Plut. 33.31, in parte ripresa da Cursi, La scrittura e i libri del Boccaccio, p. 18. 
scienza del valore insito nell'atto dello scrivere, si pone su un piano per certi versi analogo a quello dei suoi immediati successori Petrarca e Boccaccio.

\section{Bibliografia}

\section{Fonti}

Francesco da Barberino, I Documenti d'Amore secondo i manoscritti originali, I-IV, a cura di F. Egidi, Roma 1902-1927.

Francesco da Barberino, I Documenti d'Amore / Documenta Amoris, a cura di M. Albertazzi, 2 voll., Lavis $2011^{2}$ [l ed. 2008].

Francesco da Barberino, Reggimento e costumi di donna, a cura di G.E. Sansone, Torino 1957.

\section{Studi critici}

L. Azzetta, Tra gli amici e i cultori di Dante: documenti per Francesco da Barberino, Lapo Gianni, Andrea Lancia, in Per beneficio e concordia di studio. Studi danteschi offerti a Enrico Malato per i suoi ottant'anni, a cura di A. Mazzucchi, Cittadella 2015, pp. 61-71.

A. Bartoli Langeli, Autografia e paleografia, in «Di mano propria». Gli autografi dei letterati italiani. Atti del convegno (Forlì, 24-27 novembre 2008), a cura di G. Baldassarri, M. Motolese, P. Procaccioli, Roma 2010, pp. 41-60.

L. Battaglia Ricci, Comporre il testo. Elaborazione e tradizione, in Intorno al testo: tipologie del corredo esegetico e soluzioni editoriali. Atti del Convegno (Urbino, 1-3 ottobre 2001), Roma 2003, pp. 21-40.

L. Battaglia Ricci, Edizioni d'autore, copie di lavoro, interventi di autoesegesi: testimonianze trecentesche, in «Di mano propria». Gli autografi dei letterati italiani. Atti del convegno (Forlì, 24-27 novembre 2008), a cura di G. Baldassarri, M. Motolese, P. Procaccioli, Roma 2010, pp. 123-157.

F.M. Bertolo, Apocalissi figurata. Per l'interpretazione del testo allegorico in appendice all'Officiolum' ritrovato di F. da B., in «Studi (e testi) italiani», 7 (2005), pp. 55-64.

F.M. Bertolo, T. Nocita, L'“Officiolum' ritrovato di F. da B. In margine ad una allegoria figurata inedita della prima metà del Trecento, in «Filologia e critica», 31 (2006), 1, pp. 106-117.

M. Boskovits, The Fourteenth Century. The Painters of the Miniaturist Tendency, Firenze 1984.

I. Ceccherini, Teaching, Function and Social Diffusion of Writing in Thirteenth-and FourteenthCentury Florence, in Teaching Writing, Learning to Write. Proceedings of the XVIth Colloquium of the Comité International de Paléographie Latine, ed. By P. R. Robinson, London 2008, pp. 177-192.

M. Ciccuto, Francesco da Barberino e il suo Bildercodex, in «Letteratura e Arte», 9 (2001), pp. 83-95.

M. Ciccuto, Guinizzelli e Guittone, Barberino e Petrarca: le origini del libro volgare illustrato, in «Rivista di storia della miniatura», 1/2 (1996-1997), pp. 77-87. 
M. Ciccuto, Icone della parola. Immagine e scrittura nella letteratura delle origini, Modena 1995.

M. Cursi, La scrittura e i libri del Boccaccio, Roma 2013.

M. Cursi, Per la prima circolazione dei Rerum vulgarium fragmenta: i manoscritti antiquiores, in Storia della scrittura e altre storie, a cura di D. Bianconi, Roma 2014, pp. 225-261.

C. de La Mare, The Handwriting of Italian Humanists, I.1. Francesco Petrarca, Giovanni Boccaccio, Coluccio Salutati, Niccolò Niccoli, Poggio Bracciolini, Bartolomeo Aragazzi of Montepulciano, Sozomeno of Pistoia, Giorgio Antonio Vespucci, Oxford 1973.

T. De Robertis, Digrafia nel Trecento: Andrea Lancia e Francesco di ser Nardo da Barberino, in «Medioevo e Rinascimento», 26 (2012), pp. 221-235.

T. De Robertis, Scritture di libri scritture di notai, in «Medioevo e Rinascimento», 24 (2010), pp. 1-27.

T. De Robertis, Una mano tante scritture. Problemi di metodo nell'identificazione degli autografi, in Medieval Autograph Manuscripts. Proceedings of the XVIIth Colleque du CIPL (Ljubljana, 7-10 septermber 2010), ed. by N. Golob, Turnhout 2010, pp. 18-38.

F. Egidi, Le miniature dei codici Barberiniani dei Documenti d'Amore, in «L'arte», 5 (1902), pp. 1-20; 78-95.

M. Feo, Francesco Petrarca, in Storia della letteratura italiana, X. La tradizione dei testi, a cura di C. Ciociola, Roma 2001, pp. 277-278.

C. Giunti, L'interazione fra testo e immagini (perdute) nel «Reggimento» di Francesco da Barberino, in «Bullettino dell'Istituto Storico Italiano per il Medio Evo e Archivio Muratoriano», 104 (2002), pp. 121-144.

C. Giunti, «Né parlerai rimato». Sulla struttura formale del Reggimento e costumi di donna di Francesco da Barberino, in «Studi e problemi di critica testuale», 81 (2010), pp. 71-111.

D. Goldin, Testo e immagine nei Documenti d'Amore di Francesco da Barberino, in «Quaderni di italianistica», 1 (1980), pp. 125-138.

D. Goldin, Autotraduzione latina nei 'Documenti d'Amore' di Francesco da Barberino, in «Atti dell'Istituto Veneto di Scienze Lettere ed Arti», 133 (1975), pp. 371-392.

D. Goldin Folena, Il commento di Francesco da Barberino, in Intorno al testo. Tipologie del corredo esegetico e soluzioni editoriali. Atti del Convegno (Urbino, 1-3 ottobre 2001), Roma 2003, pp. 263-282.

O. Pächt, Der Weg von der zeichnerischen Buchillustration zur eigenständigen Zeichnung, in «Wiener Jahrbuch für Kunstgeschichte», 24 (1971), pp. 178-184.

M.C. Panzera, Per l'edizione critica dei «Documenti d'Amore» di Francesco da Barberino, in «Studi mediolatini e volgari», 40 (1994), pp. 91-118.

A. Petrucci, Dalla minuta al manoscritto d'autore, in Lo spazio letterario del Medioevo, 1. Il Medioevo latino, I. La produzione del testo, Roma 1992, pp. 353-372.

A. Petrucci, Funzione della scrittura e terminologia paleografica, in Palaeographica, diplomatica et archivistica, 2 voll., Roma 1979, I, pp. 1-30.

A. Petrucci, La scrittura del testo, in Letteratura italiana, IV. L'interpretazione, a cura di A. Asor Rosa, Torino 1985, pp. 289-290.

A. Petrucci, La scrittura di Francesco Petrarca, Città del Vaticano 1967.

A. Petrucci, Libro e scrittura in Francesco Petrarca, in Libri, scrittura e pubblico nel Rinascimento, a cura di A. Petrucci, Roma-Bari 1979, pp. 5-20.

A. Petrucci, Minima barberina. I. Note sugli autografi dei «Documenti d'Amore», in Miscellanea di studi in onore di Aurelio Roncaglia a cinquant'anni dalla sua laurea, 4 voll., III, Modena 1989, pp. 1005-1009. 
A. Petrucci, Minuta, autografo, libro d'autore, in Il libro e il testo. Atti del Convegno internazionale (Urbino, 20-23 settembre 1982), a cura di C. Questa, R. Raffaelli, Urbino 1984, pp. 397-414.

A. Petrucci, Modello notarile e testualità, in Il notariato nella civiltà toscana. Atti del Convegno (Roma, Consiglio Nazionale del Notariato, maggio 1981), Roma 1985, pp. 138-139.

A. Petrucci, Scrivere il testo, in La critica del testo. Problemi di metodo ed esperienze di lavoro, Roma 1985, pp. 209-227.

A. Petrucci, Spazi dei testi e strategie petrarchesche, in La parola scritta e le sue grazie. A proposito della mostra «Petrarca nel tempo». Discorsi di Alberto Asor Rosa et al., Pontedera 2006, pp. 45-56.

A. Petrucci, Storia e geografia delle culture scritte (dal secolo XI al XVIII), in Letteratura italiana. Storia e geografia, II/2. L'età moderna, a cura di A. Asor Rosa, Torino 1988, pp. 1226-1227.

G. Pozzi, Dall'orlo del visibile parlare, in Visibile parlare. Le scritture esposte nei volgari italiani dal Medioevo al Rinascimento. Atti del Convegno Internazionale di Studi (Cassino-Montecassino, 26-28 ottobre 1992), a cura di C. Ciociola, Napoli 1997, pp. 15-41.

L. Rockinger, Briefsteller und Formelbücher des eilften bis vierzehnten Jahrhunderts, Munchen 1864.

M. Signorini, Fortuna del "modello-libro" Canzoniere, in «Critica del testo», 6/1 (2003), pp. 133-154.

M. Signorini, La scrittura libraria di Francesco Petrarca: terminologia, fortuna, in «Studi medievali», 48/2 (2007), pp.839-862.

P. Supino Martini, Per la storia della semigotica, in «Scrittura e civiltà», 22 (1998), pp. 249-264.

P. Supino Martini, Per la tradizione manoscritta dei «Documenti d'Amore» di Francesco da Barberino, in «Studi medievali», s. III, 37 (1996), pp. 945-954.

K. Sutton, The lost 'Officiolum' of Francesco da Barberino rediscovered, in «The Burlington Magazine», 147 (2005), pp. 152-164.

S. Zamponi, Il libro del Canzoniere: modelli, strutture, funzioni, in Rerum Vulgarium Fragmenta. Codice Vat. Lat. 3195. Commentario all'edizione in facsimile, a cura di G. Belloi, F. Brugnolo, H. W. Storey, S. Zamponi, Roma-Padova $\langle$ Y $\rangle$ 2004, pp. 13-72.

S. Zamponi, M. Pantarotto, A. Tomiello, La stratigrafia dello Zibaldone e della Miscellanea laurenziani, in Gli Zibaldoni di Boccaccio. Memoria, scrittura, riscrittura. Atti del Seminario internazionale (Certaldo-Firenze, 26-28 aprile 1996), a cura di M. Picone, C. Cazalé Bérard, pp. 181-258. 